\title{
Overexpression of ZNF217 in glioblastoma contributes to the maintenance of glioma stem cells regulated by hypoxia-inducible factors
}

\author{
Xing-gang Mao ${ }^{1,6}$, Ming Yan², ${ }^{2,}$ Xiao-yan Xue ${ }^{3,6}$, Xiang Zhang ${ }^{1}$, Hong-gang Ren ${ }^{4}$, Geng Guo ${ }^{1,5}$, Peng Wang ${ }^{1}$, \\ Wei Zhang ${ }^{1}$ and Jun-li Huo ${ }^{1}$
}

\begin{abstract}
Glioblastoma multiforme (GBM) is the most aggressive and common kind of primary brain tumor in adults, and is thought to be driven by a subpopulation of glioma stem cells (GSCs). GSCs reside in a specialized hypoxic niche, which can regulate the tumorigenic capacity of GSCs primarily through the hypoxia-inducible factors (HIFs), HIF1 $\alpha$ and HIF $2 \alpha$. ZNF217 is an oncogene frequently amplified in many kinds of tumors. It is associated with aggressive tumor behavior and poor clinical prognosis, but its role in gliomas is poorly known. Gene expression and copy number analysis from TCGA data reveal that ZNF217 is amplified in 32\% and overexpressed in 71.2\% of GBMs. Quantitative RT-PCR and western blotting of a cohort of glioma samples showed that ZNF217 was highly expressed in gliomas and increased with tumor grade. Analysis of a molecular database demonstrated that ZNF217 expression correlated with poor survival of glioma patients. Investigation of ZNF217 expression in GSCs, non-GSCs and normal neural stem cells (NSCs) indicated that ZNF217 was more highly expressed in GSCs than in non-GSCs and NSCs. Knockdown of ZNF217 in GSCs by smallinterfering RNA (siRNA) inhibited their growth and promoted their differentiation. Interestingly, ZNF217 was upregulated in GSCs and the GBM cell line U87 when exposed to the hypoxic environment of $1 \%$ oxygen. Knockdown of either HIF1 $\alpha$ or HIF2 $\alpha$, which has a central role in the hypoxia-induced responses of these cells, inhibited ZNF217 expression. In addition, ZNF217 upregulation was compromised under hypoxia in U87 and GSCs when either HIF1 $\alpha$ or HIF2 $\alpha$ was targeted by siRNA. HIF2 $\alpha$ knockdown inhibited ZNF217 expression more efficiently in both normoxia and hypoxia than HIF1 $\alpha$ knockdown. Therefore, ZNF217 is overexpressed in GBMs and contributes to the maintenance of GSCs, which is regulated by HIFs released by the hypoxic environment of the tumor.

Laboratory Investigation (2011) 91, 1068-1078; doi:10.1038/labinvest.2011.56; published online 11 April 2011
\end{abstract}

KEYWORDS: cancer stem cell; CD133; HIF1 $\alpha$; HIF2 $\alpha$; hypoxia

Brain tumors have one of the poorest outcomes of all human cancers. ${ }^{1}$ Glioblastoma multiforme (GBM, World Health Organization grade IV glioma) is the most aggressive and, unfortunately, most common type of primary brain tumors in adults with a median survival of approximately 1 year, ${ }^{2}$ despite advances in surgery and adjuvant therapies. The failure of current treatments for GBMs primarily attributes to their invasiveness and resistance to chemo- and radiotherapies, resulting in frequent relapse or progression.
Currently, the mechanisms underlying GBM tumorigenesis are not fully known. The cancer stem cell (CSC) hypothesis suggests that GBM is driven by a subpopulation of glioma stem cells (GSCs), which also contribute to their resistance to therapy. ${ }^{3-7}$ Therefore, it is important for both cancer research and clinical practice to further explore the intrinsic molecular basis behind the tumorigenicity of GSCs. Great efforts have been made to understand the mechanisms underlying the stem cell properties and tumorigenicity of GSCs. It has been

\footnotetext{
'Department of Neurosurgery, Xijing Hospital, Fourth Military Medical University, Xi'an, Shaanxi Province, China; ${ }^{2}$ Department of Orthopaedic Surgery, Xijing Hospital, Institute of Orthopaedics and Traumatology of PLA of China, The Fourth Military Medical University, Xi'an, Shaanxi Province, China; ${ }^{3}$ Department of Pharmacology, School of Pharmacy, The Fourth Military Medical University, Xi'an, Shaanxi Province, China; ${ }^{4}$ Department of Neurosurgery, Linfen People's Hospital, Linfen, Shaanxi Province, China and ${ }^{5}$ Department of Neurosurgery, The First Hospital, Shanxi Medical University, Taiyuan, Shanxi, China

Correspondence: Dr X Zhang, MD, PhD, Department of Neurosurgery, Xijing Hospital, Fourth Military Medical University, No. 17 Changle West Road, Xi'an, Shaanxi Province 710032, China.

E-mail: xzhang@fmmu.edu.cn

${ }^{6}$ These authors contributed equally to this work.

Received 29 November 2010; revised 10 February 2011; accepted 13 February 2011
} 
well established that GSCs are enriched in vascular and perinecrotic/hypoxic niches, where hypoxia is a critical component of the microenvironment. ${ }^{8-11}$ It has been reported that hypoxia promotes reprogramming of many cell types to a more primitive state, including neural stem cells $(\mathrm{NSCs})^{12}$ and, more importantly, CSCs. ${ }^{13,14}$ In addition, hypoxia also contributes to the aggressiveness and resistance of cancers to therapy. ${ }^{15,16}$ Hypoxia affects many key processes of cells through the coordinated regulation of a large number of genes. Hypoxia-inducible factors (HIFs), including HIF1 $\alpha$ and HIF $2 \alpha$, are transcription factors regulated by oxygen levels that have a central role in the hypoxia-induced responses of cells. ${ }^{17-19}$

ZNF217, a candidate oncogene found on chromosome $20 \mathrm{q} 13.2$, is frequently amplified in many tumors, such as breast and ovarian cancers. ${ }^{20-25}$ In addition, ZNF217 is associated with aggressive tumor behavior and poor clinical prognosis; however, its role in gliomas is poorly understood. Genome-wide chromatin immunoprecipitation (ChIP) and transcript profiling elucidated that ZNF217 can regulate a large number of genes, while acting as a transcription factor, together with other co-factors, ${ }^{26,27}$ indicating that it may have a wide range of biological functions in tumor cells. One potential function of ZNF217 is that it may repress differentiation and contribute to the maintenance of CSCs. ${ }^{26}$ Herein, we show that ZNF217 is highly expressed in GBMs and its elevated expression is associated with poor prognosis. In addition, it also contributes to the maintenance of GSCs by inhibiting their differentiation. Interestingly, ZNF217 is upregulated under hypoxia and may be a downstream target of HIFs, implying that it is involved in the hypoxia-induced maintenance of GSCs.

\section{MATERIALS AND METHODS Glioma Samples}

The following brain tumor samples used for quantitative real-time PCR (qPCR) and western blotting were provided by the Department of Neurosurgery, Xijing Hospital, the Fourth Military Medical University: 31 GBMs (M/F ratio: 19/12; median age: 54.0 (24-68)), 26 grade II gliomas (14 astrocytoma and 12 oligodendroglioma; M/F ratio: 12/14; median age: $46.6(27-64))$ and 27 grade III gliomas (15 anaplastic astrocytoma and 11 anaplastic oligodendroglioma, M/F ratio: 15/12; median age: 48.1 (24-65)). Tumors were histopathologically classified according to the WHO classification. Informed consent was obtained from each patient, and experiments were approved by the local ethics committee.

\section{Culture of Primary GSCs and NSCs}

GSCs were cultured as described previously. ${ }^{28}$ The study protocol was approved by Institutional Review Committee (IRB) of Xijing Hospital of the Fourth Military Medical University and written informed consent was obtained from patients. Briefly, samples were dissociated to a single cell suspension using a fire-polished Pasteur pipette and cultured in serum-free medium (SFM) consisting of DMEM-F12 medium, EGF (20 ng/ml; Invitrogen, Carlsbad, CA, USA), bFGF (20 ng/ml; Invitrogen) and B27 (1:50; Invitrogen), or in $10 \%$ serum-containing medium. Two human fetal cortical NSC cell lines were isolated from spontaneous aborted fetuses (8-12 weeks), which were dissociated into single cells as described above and then cultured in SFM.

For the differentiation assay of GSCs and NSCs, neural spheres were subjected to forced differentiation in $10 \%$ serum-containing medium without growth factors for 14 days.

In order to induce hypoxia, cells were cultured in a sealed Modular Incubator Chamber (Billups-Rothenberg, Del Mar, CA, USA) flushed with $1 \% \mathrm{O}_{2}, 5 \% \mathrm{CO}_{2}$ and $94 \% \mathrm{~N}_{2}$ and incubated at $37^{\circ} \mathrm{C}$ for $24 \mathrm{~h}$.

\section{Immunofluorescence}

Immunofluorescence was performed on GSCs as described previously. ${ }^{28}$ Briefly, tumor spheres were dissociated into single cells and plated onto poly-L-lysine-coated glass coverslips in SFM for $4 \mathrm{~h}$, fixed with $4 \%$ paraformaldehyde and incubated with the following primary antibodies overnight at $4{ }^{\circ} \mathrm{C}$ : Nestin $(1: 100,10 \mathrm{C} 2$, mouse monoclonal; Abcam, Cambridge, MA, USA), Tuj1 (1:500, mouse monoclonal IgG1; Abcam), GFAP (1:5000, rabbit polyclonal; Abcam) and Ki67 (1:100, rabbit monoclonal; Abcam). Appropriate secondary antibodies (Texas Red goat anti-rabbit, and Alexa 488 goat anti-mouse, Molecular Probes, Invitrogen) were used. Counterstaining with Hoeschst 33342 (Sigma, St Louis, MO, USA) was additionally performed to permit counting of cell nuclei.

\section{Quantitative Real-Time PCR}

RNA was extracted from cultured cells and brain tumor tissues using Trizol Reagent (Invitrogen). The extracted RNA was then reverse transcribed into cDNA and qPCR analysis was performed on an ABI7700 using SYBR Green PCR Core Reagents in $20 \mu \mathrm{l}$ volume (Applied Biosystems, Warrington, UK). The following primers were used for qPCR analysis: ZNF217 forward $5^{\prime}$-ATTCTCCAAATGTGCTGACTGTTC- $3^{\prime}$ and reverse: $5^{\prime}$-TGCCATGCTGTTAGATAAGTGTTG- $3^{\prime}$; HIF $1 \alpha$ forward $5^{\prime}$-TTCCAGTTACGTTCCTTCGATCA- $3^{\prime}$ and reverse: $5^{\prime}$-TTTGAGGACTTGCGCTTTCA- $3^{\prime}$; HIF2 $\alpha$ forward $5^{\prime}$-GTGCTCCCACGGCCTGTA- $3^{\prime}$ and reverse: $5^{\prime}$-TTGTCAC ACCTATGGCATATCACA- $3^{\prime} ; \beta$-actin forward $5^{\prime}$-CCCAGCA CAATGAAGATCAA- $3^{\prime}$ and reverse: $5^{\prime}$-GATCCACACGGAG TACTTG-3' ${ }^{\prime}$. qPCR using water instead of template was used as negative controls. All samples were assayed in triplicate and the relative amount of target transcripts normalized to the number of human $\beta$-actin transcripts found in the same sample. Specificity was verified by melting curve analysis and agarose gel electrophoresis. Relative fold changes were calculated using the $\Delta \Delta \mathrm{C}_{\mathrm{t}}$ method by using the threshold cycle values of each sample.

\section{Western Blotting}

Cultured cells were lysed in SDS sample buffer, and $30 \mu \mathrm{g}$ proteins were run on $6 \%$ SDS-PAGE gel and transferred to a 
nitrocellulose membrane. Blots were blocked in PBS containing 5\% non-fat dry milk powder and incubated overnight at $4{ }^{\circ} \mathrm{C}$ with primary antibody of $\mathrm{CD} 133$ (1:500; Abcam), HIF $1 \alpha$ (1:1000; BD Biosciences, San Jose, CA, USA), HIF2 $\alpha$ (1:500; Novus Biologicals, Littleton, CO, USA), ZNF217 (1:500; Abcam) or GAPDH (1:500,000; Abcam). Blots were then washed with PBS containing $0.1 \%$ Tween 20 (PBST) and incubated in secondary antibodies coupled to peroxidase. After washing in PBST, blots were developed with chemiluminescence according to the manufacturer's instructions (enhanced chemiluminescence, Amersham Biosciences, GE Healthcare, France). All western blot analyses were done in duplicate.

\section{Small-Interfering RNA Transfection}

The tumor spheres were dissociated into single cells and plated on poly-L-ornithine $(50 \mathrm{mg} / \mathrm{ml}, \mathrm{P} 3655$, Sigma Chemical)-coated wells (50000 cells per 24-well platewell) to attach for $24 \mathrm{~h}$. Then the cells were transfected by Stealth small-interfering RNAs (siRNA) targeting ZNF217, HIF1 $\alpha$, HIF $2 \alpha$ or non-targeting RNA sequence (Invitrogen Lifetechnologies, USA) with Oligofectamine ${ }^{\mathrm{TM}}$ RNAiMAX reagent according to the manufacturers' protocol (Invitrogen Lifetechnologies). After $24 \mathrm{~h}$ of incubation, the cells were washed and detached from the ornithine-coated wells and placed back to SFM medium. The efficiency of siRNA interference was evaluated by qPCR after $72 \mathrm{~h}$ of transfection.

To evaluate the proliferation potential of cells after RNA interference, cells were plated on poly-L-ornithine coverslips, transfected with siRNA and stained for Ki67. For growth kinetics, cells were transfected with siRNA, collected and then used to perform growth curve analysis up to 6 days after transfection. Briefly, the collected cells were plated in 96-well plates in $0.2 \mathrm{ml}$ volumes of SFM medium, at a density of 2000 cells/well. Tumor spheres were collected from six wells every 2 days after plating and dissociated to get single cells as described above to assess the total number of cells. All results are averages of at least three parallel siRNA-transfected cell pools.

\section{Statistical Analysis}

Statistical analyses were performed using Student's $t$-tests and one-way analysis of variance with least squared difference post hoc tests, as appropriate. All $P$-values are two tailed. A value of $P<0.05$ was considered statistically significant. Statistical analyses were done using SPSS v.13.0.0 (SPSS, Chicago, IL, USA).

\section{RESULTS}

\section{ZNF217 Is Overexpressed and Frequently Amplified in GBMs}

First, we used the TCGA data $\operatorname{set}^{29}$ to investigate the expression and copy number of ZNF217 in GBMs. In total, ZNF217 is overexpressed in $71.2 \%$ of the GBMs (more than twofold increase). Strikingly, $57.2 \%$ of the GBM samples showed a fourfold increase of ZNF217 expression compared with normal brain tissues, while none of the samples showed a significantly decreased expression (Figure 1a and b). Copy number analysis demonstrated that $32.0 \%$ of the GBMs exhibited gene amplification, while only one sample exhibited decreased copy number of ZNF217 (Figure 1a and b). ZNF217 amplification is more frequently found in the samples where it is overexpressed $(46.6 \%$ in ZNF217-overexpressed GBMs vs $17.0 \%$ in non-overexpressed GBMs; $P<0.001, \chi^{2}$ analysis; Figure 1c), suggesting that the overexpression is primarily due to gene amplification. Nonetheless, $53.4 \%$ of the GBM samples with ZNF217 overexpression did not show gene amplification, indicating that other unknown mechanisms also contribute to its increased expression.

\section{ZNF217 Is Highly Expressed in Gliomas and Specifically Activated in GBMs}

To further characterize the expression of ZNF217 in gliomas, we performed qPCR on a cohort of normal and glioma samples, including 5 normal brain samples, 14 astrocytomas (A, grade II), 12 oligodendrogliomas (O, grade II), 15 anaplastic astrocytomas (AA, grade III), 11 anaplastic oligodendrogliomas (AO, grade III) and 31 GBMs. In total, all grades of gliomas, including GBMs, grades II and III astrocytomas and oligodendrogliomas, showed higher levels of ZNF217 compared with normal tissues (Figure 2a and b), with mRNA levels increasing with tumor grade $(P<0.001)$. Compared with normal tissues, the expression of ZNF217 was increased by $2.07 \pm 0.89$-, $2.42 \pm 0.90$ - and $3.53 \pm 1.90$ fold in grades II and III astrocytomas and GBMs, respectively, and increased by $1.58 \pm 0.60$ - and $1.99 \pm 0.70$-fold in grades II and III oligodendrogliomas, respectively. Interestingly, although not statistically significant, astrocytomas expressed higher levels of ZNF217 than oligodendrogliomas (Figure 2a and b). Noticeably, ZNF217 was more significantly overexpressed in GBMs than in normal and low-grade gliomas $(P<0.05)$ except for anaplastic astrocytomas $(P=0.082)$. To further confirm the increased expression of ZNF217 in gliomas, proteins were extracted from two normal brain samples, five grade II astrocytomas, five grade III astrocytomas and five GBM samples. Western blotting analysis demonstrated that the ZNF217 protein was detected in normal and glioma tissues and was more highly expressed in gliomas (Figure 2c).

\section{Elevated ZNF217 Expression Is Associated with Poor Survival of Glioma Patients}

ZNF217 expression and gene copy number analysis demonstrate that ZNF217 is overexpressed in gliomas, especially in GBMs, implying its expression may be associated with clinical outcomes of glioma patients. To investigate whether targeting ZNF217 might have a therapeutic benefit for the glioma patients, we utilized the REMBRANDT (Repository of Molecular Brain Neoplasia Data) database of the National 


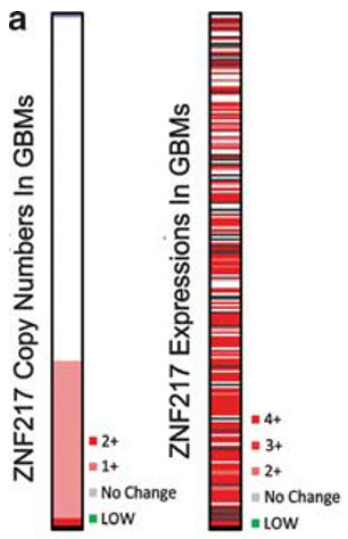

C

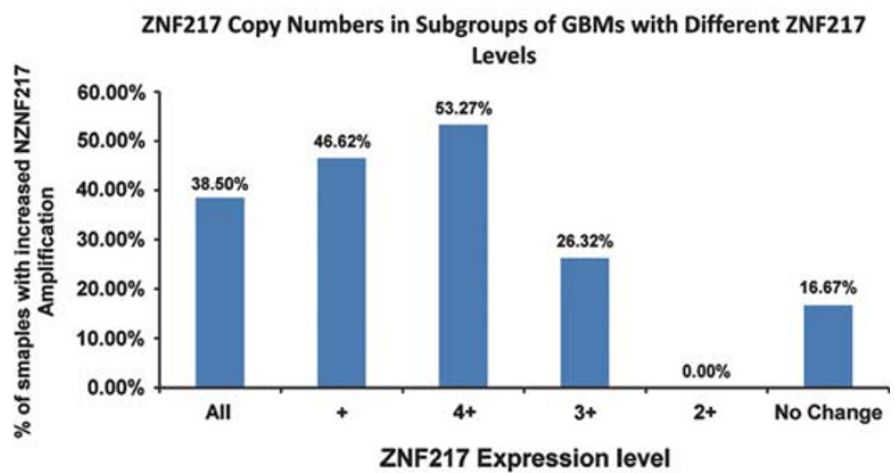

b ZNF217 Copy Numbers in GBMs

ZNF217 Expressions in GBMs
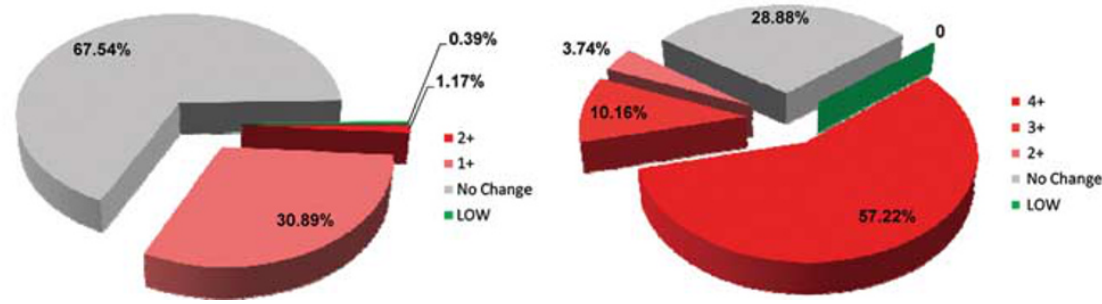

Figure 1 Overexpression and amplification of ZNF217 in GBMs. (a, b) TCGA database analysis demonstrated that the ZNF217 gene is overexpressed in $71.2 \%$ (more than twofold increase compared with normal tissues) and amplified in 32\% of GBMs. (c) Relationship between amplification and overexpression of ZNF271 in GBMs. ZNF217 amplification is more frequently found in ZNF217-overexpressing GBMs (46.6\%) than in non-overexpressing GBMs (16.67\%; $P<0.001, \chi^{2}$ analysis). 1-4 + indicate gained copy numbers or increased fold-increase of ZNF217 expression in GBMs compared with normal tissues.

Cancer Institute (http://caintegrator-info.nci.nih.gov/rembrant). The data were analyzed to determine the survival of 343 glioma patients with intermediate, low or high expression of ZNF217. There were 214 patients with more than twofold ZNF217 upregulation and only 4 patients with greater than twofold ZNF217 downregulation. Importantly, we found a significant decrease in the probability of survival with elevated ZNF217 $(P<0.001$, Figure 3$)$. Therefore, these data suggest that ZNF217 expression affects patient outcome and supports a specific and important role for ZNF217 in gliomas.

\section{GSCs Are Enriched in ZNF217}

It has been suggested that gliomas are driven by a subpopulation of GSCs. GSCs share similar properties to normal NSCs, and cause xenografts more closely resembling primary gliomas than serum-cultured non-CSCs when injected into brains of immunocompromised mice. ${ }^{30}$ Our results demonstrate that elevated ZNF217 expression in gliomas is associated with poor prognosis. We further investigated if ZNF217 would be preferentially expressed in GSCs and contribute to the tumorigenicity of gliomas fueled by GSCs. We compared the expressions of ZNF217 in GSCs and nonGSCs. Seven glioma-derived neurosphere cell lines cultured in SFM, which are enriched for stem-like cells, ${ }^{28,30,31}$ were used as GSCs. In addition, $10 \%$ serum-cultured primary glioma cells and the GBM cell lines U87, U251 and A172 were used as non-GSCs ${ }^{30}$ and two normal NSC cell lines from human fetal brains cultured in SFM were used as normal controls. Immunofluorescence and western blotting demonstrated that the GSC markers Nestin, CD15, CD133 and OLIG2 were expressed in GSCs, but non-expressed or lowly expressed in serum-cultured GBM cell lines and primary non-GSCs. This indicates that SFM-cultured glioma spheres were enriched in GSCs, while serum-cultured primary glioma cells and GBM cell lines contained less or no GSCs (Figure 4a and b).

qPCR showed that ZNF217 was expressed in both GSCs and normal NSCs, but at higher levels of expression in GSCs (Figure 4c and d). Importantly, both GSCs and NSCs expressed higher levels of ZNF217 than non-GSCs (Figure 4c and d). These results indicate that stem cells, especially GSCs, are enriched in ZNF217. In support of this hypothesis, GSCs were subjected to forced differentiation in $10 \%$ serum medium without growth factors for 2 weeks. Western blotting showed that the stem cell marker CD133 was downregulated and glial marker GFAP was upregulated in differentiated GSCs (Figure 4e). Furthermore, qPCR demonstrated that ZNF217 was downregulated in six of the seven GSC cell lines by $36-74 \%(55 \pm 16 \%)$ after differentiation (Figure $4 \mathrm{f}$ ), 

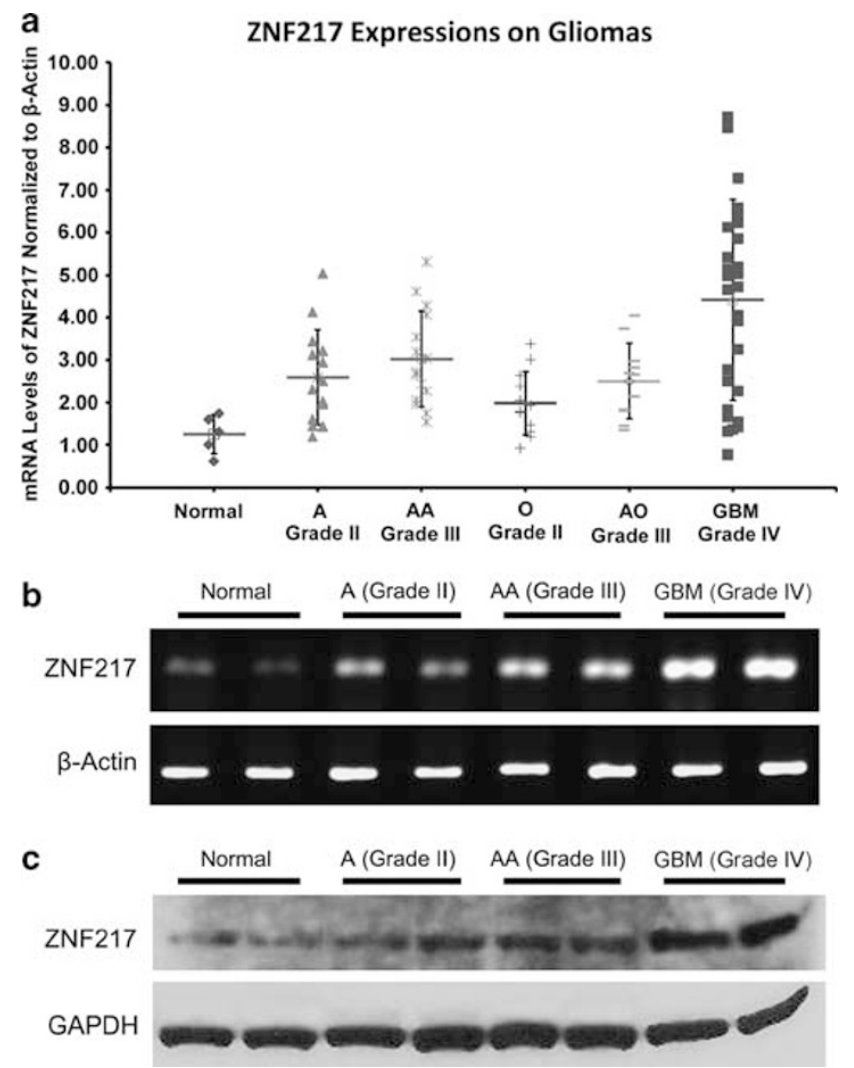

Figure 2 ZNF217 expression in gliomas, as compared with normal brain tissue. (a) qPCR analysis of ZNF217 expression in astrocytomas (A), anaplastic astrocytomas (AA), oligodendrogliomas $(\mathrm{O})$, anaplastic oligodendrogliomas (AO) and GBMs reveals increased expression in all types of gliomas, especially GBMs, compared with normal brain tissue. GBMs expressed significantly higher levels of ZNF217 than normal and lowgrade gliomas ( $\mathrm{A}, \mathrm{O}$ and $\mathrm{OA} ; \mathrm{P}<0.05)$. (b, c) Representative RT-PCR and western blots showing ZNF217 is more highly expressed in gliomas than in normal and low-grade glioma tissues. $\beta$-Actin and GAPDH were used as loading controls for RT-PCR and western blotting, respectively.

further supporting the notion that stem-like cells are enriched in ZNF217.

\section{ZNF217 Promotes Proliferation of GSCs and Contributes to Their Maintenance}

To investigate the potential role of ZNF217 in GSCs, we used siRNA knockdown of ZNF217 in GSC11 and GSC5. qPCR revealed that ZNF217 was downregulated by 63 and $72 \%$ in GSC11 and GSC5, respectively, compared with non-targeting controls (Figure 5a). The proliferation of GSC11 and GSC5 was significantly decreased after ZNF217 knockdown compared with non-targeting controls, as estimated by Ki67 staining and growth curve analysis (Figure $5 b-d$ ). Furthermore, we estimated the percentage of Nestin-positive, GFAPpositive and Tuj1-positive cells in GSCs treated with ZNF217 siRNA or non-targeting siRNA. The results showed that ZNF217 knockdown decreased the percentage of Nestinpositive stem-like cells, while it increased the percentage of the more differentiated GFAP-positive and Tuj1-positive cells (Figure $5 \mathrm{c}-\mathrm{e}$ ), indicating that ZNF217 has a role in maintaining the stem cell characteristics and its downregulation promoted the differentiation of GSCs.

\section{ZNF217 Is Upregulated in GSCs and GBM Cell Lines in Hypoxic Conditions}

In recent years, it has been established that hypoxia is an important factor for the GSC niche and can promote their tumorigenic capacity and clonogenicity. ${ }^{8,9,32}$ As we have already shown that ZNF217 is important for the maintenance of GSCs, we then decided to determine whether it is involved in the hypoxia-regulated maintenance of GSCs. Therefore, we investigated whether ZNF217 would be regulated by hypoxia.

U87 and the seven GSC cell lines were cultured under hypoxic $\left(1 \% \mathrm{O}_{2}\right)$ or normal $\left(20 \% \mathrm{O}_{2}\right)$ conditions for $24 \mathrm{~h}$. Western blotting showed that HIF $1 \alpha$ and HIF $2 \alpha$ and the stem cell marker CD133 were increased in U87 and GSC cell lines (Figure 6a) under hypoxia. ZNF217 mRNA showed a 2.3-fold increase in U87 and 1.2-8.7-fold (3.4 \pm 2.5$)$ increase in the GSC cell lines under hypoxia (Figure $6 \mathrm{~b}$ ). Western blotting further demonstrated that ZNF217 protein was upregulated under hypoxia in U87 and GSCs (Figure 6a). These results suggested that ZNF217 was increased under hypoxia in both serum-cultured non-GSCs and SFM-cultured GSCs, which may contribute to the increase of stem-like cells in hypoxic conditions.

\section{ZNF217 Is Regulated by HIF1 $\alpha$ and HIF2 $\alpha$}

As HIF $1 \alpha$ and HIF $2 \alpha$ have a central role in hypoxia-induced responses of GSCs, ${ }^{9,19,32,33}$ we explored whether increased ZNF217 levels under hypoxic conditions are regulated by HIFs. First, to find potential associations between HIFs and ZNF217, we used qPCR to detect HIF $1 \alpha$ and HIF2 $\alpha$ in the cohort of GBM samples. Correlation analysis revealed that ZNF217 was significantly correlated with both HIF1 $\alpha$ $\left(R^{2}=0.32, P<0.001\right)$ and HIF2 $\alpha\left(R^{2}=0.23, P<0.01\right.$; Figure $6 \mathrm{c}$ and d), implying potential interactions between ZNF217 and HIFs. To examine whether HIF $1 \alpha$ and HIF $2 \alpha$ would regulate ZNF217 expression, we performed siRNA knockdown of HIF $1 \alpha$ and HIF $2 \alpha$ in U87 and two GSC cell lines. The efficiencies of HIF $1 \alpha$ and HIF $2 \alpha$ knockdown, as determined by qPCR, were shown in Figure 6e and f. qPCR analysis revealed that knockdown of either HIF $1 \alpha$ or HIF $2 \alpha$ significantly repressed ZNF217 expression. Notably, knockdown of HIF2 $\alpha$ repressed ZNF217 expression more efficiently than HIF $1 \alpha$, indicating that HIF2 $\alpha$ may regulate ZNF217 more efficiently than HIF $1 \alpha$ (Figure $6 \mathrm{~g}$ ). To further confirm the regulation of ZNF217 by HIFs, U87 and GSC cells transfected with HIF $1 \alpha$, HIF $2 \alpha$ or non-targeting control siRNA were cultured under 20 or $1 \%$ oxygen conditions for $24 \mathrm{~h}$, and their effects on ZNF217 expression were determined by qPCR. Compared with non-targeting controls, HIF $1 \alpha$ and HIF2 $\alpha$ knockdowns showed compromised increases of ZNF217 under hypoxia, with HIF $2 \alpha$ knockdown 


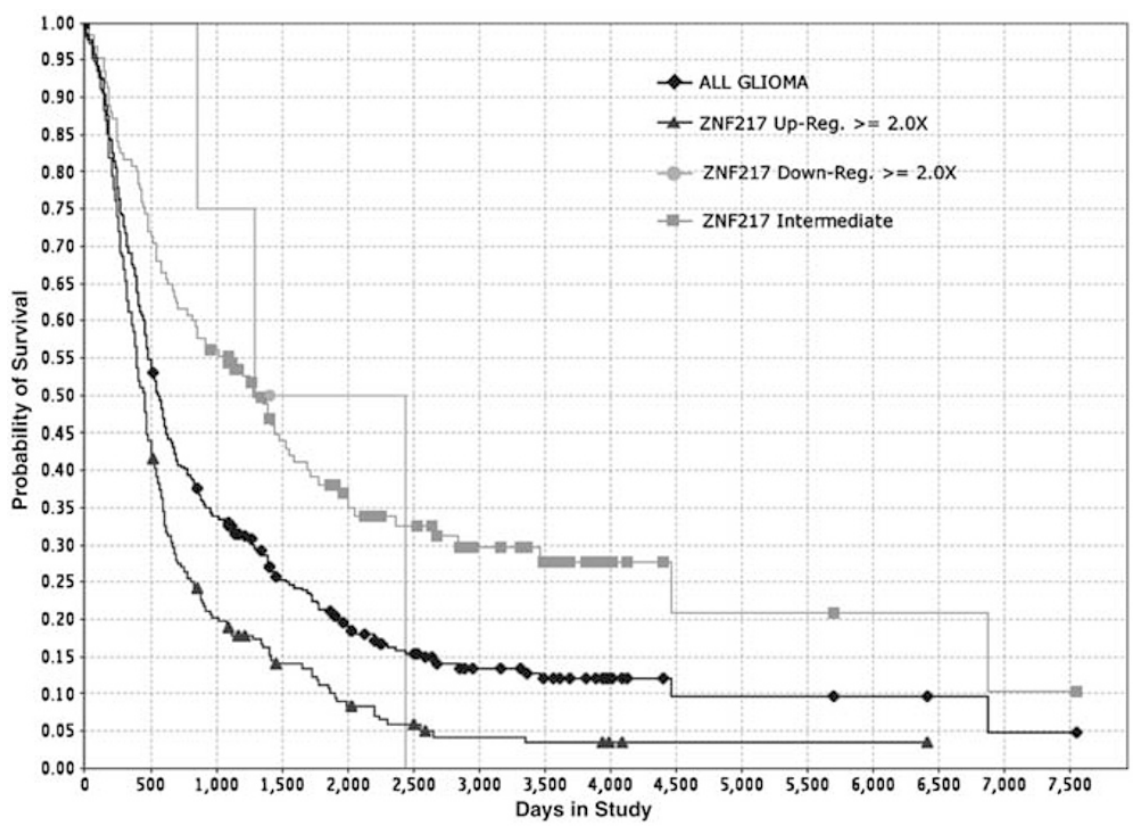

Figure 3 Kaplan-Meier survival plot for samples with differential ZNF217 gene expression. ZNF217 levels correlate with patient survival (Rembrandt database of the National Cancer Institute). There were only four patients with more than twofold ZNF217 downregulation in gliomas. $P<0.001$ with ZNF217 high vs intermediate.

more effectively repressing ZNF217 upregulation (Figure 6h). These data indicate that the increase of ZNF217 under hypoxia was regulated, at least partially, by HIF1 $\alpha$ and, more efficiently, by HIF $2 \alpha$. Together with the fact that ZNF217 contributes to the maintenance of GSCs, these data suggest that ZNF217 may contribute to increased stem cell properties of GSCs induced by hypoxia through HIF-regulated pathways.

\section{DISCUSSION}

GSCs were suggested to be the driving force of glioma progression and potential therapeutic targets. ${ }^{3,5,6}$ Unfortunately, there is a lack of specific and effective treatment targeting GSCs primarily because of an incomplete understanding of their biological features. ${ }^{34}$ For example, studies have suggested that CD133 is a marker of GSCs, while other recent studies have also suggested the existence of CD133-negative GSCs. ${ }^{35,36}$ Similarly, another stem cell marker CD15/SSEA-1 does not enrich for a population of GSCs in every tumor. ${ }^{28,37}$ Recently, it has been elucidated that GBM can be classified into several groups according to their gene profiles, which have quite different biological features and therapeutic responses. The existence of different GBM types explains partially why no universal and specific markers for all GSCs have been identified to date. ${ }^{38,39}$ Recent reports elucidated that GSCs were heterogeneous populations and CD133-positive GSCs only represent a portion of GSCs. ${ }^{40}$ Therefore, it would be important to further elucidate the characteristics of GSCs and clarify mechanisms underlining their tumorigenicity.

Great efforts have been made to elucidate the mechanisms controlling GSC maintenance and proliferation. Many genes and pathways were reported to promote the proliferation and inhibit the differentiation of GSCs, such as Wnt, Notch, SHH and BMI1. ${ }^{6,41-45}$ These pathways are also involved in the proliferation of normal NSCs, but aberrantly regulated in GSCs resulting in their uncontrolled proliferation and resistance to differentiation. ${ }^{6,46}$ Therefore, the promotion of GSC differentiation has been suggested as a potential strategy to eliminate GSCs and ultimately inhibit glioma growth.

ZNF217 is an oncogene amplified and overexpressed in many tumors, such as ovarian, breast, pancreas and colon cancers. ${ }^{20,22-24}$ It has been suggested that ZNF217 may repress differentiation by acting as a transcriptional repressor and cooperating with other transcription factors to influence a large number of downstream genes. ${ }^{26}$ This suggests that ZNF217 may have a role in maintaining stem cells and inhibiting differentiation. Therefore, we investigated if ZNF217 would have a role in the maintenance of GSCs and tumorigenicity of gliomas. We first show that ZNF217 is amplified in $32.0 \%$ and overexpressed in $71.2 \%$ of GBMs. Importantly, ZNF217 levels are increased with tumor grade and ZNF217 is specifically activated in GBMs. In addition, elevated ZNF217 is associated with poor outcomes of glioma patients. These results imply that ZNF217 may serve as an oncogene to promote the tumorigenicity of gliomas. Analysis of ZNF217 expression in GSCs, NSCs and non-GSCs demonstrates that ZNF217 is several-fold more highly expressed in GSCs than in serum-cultured cells that are further along the differentiation pathway. Consistently, forced differentiation of GSCs in serum-containing medium downregulated ZNF217, suggesting that GSCs are enriched in ZNF217. To directly examine if ZNF217 would affect the stem cell properties and 


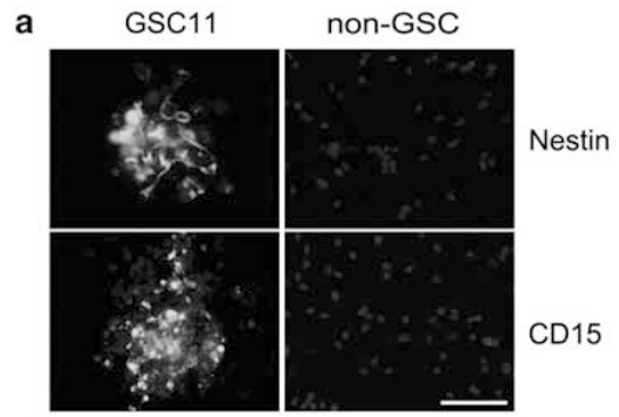

b
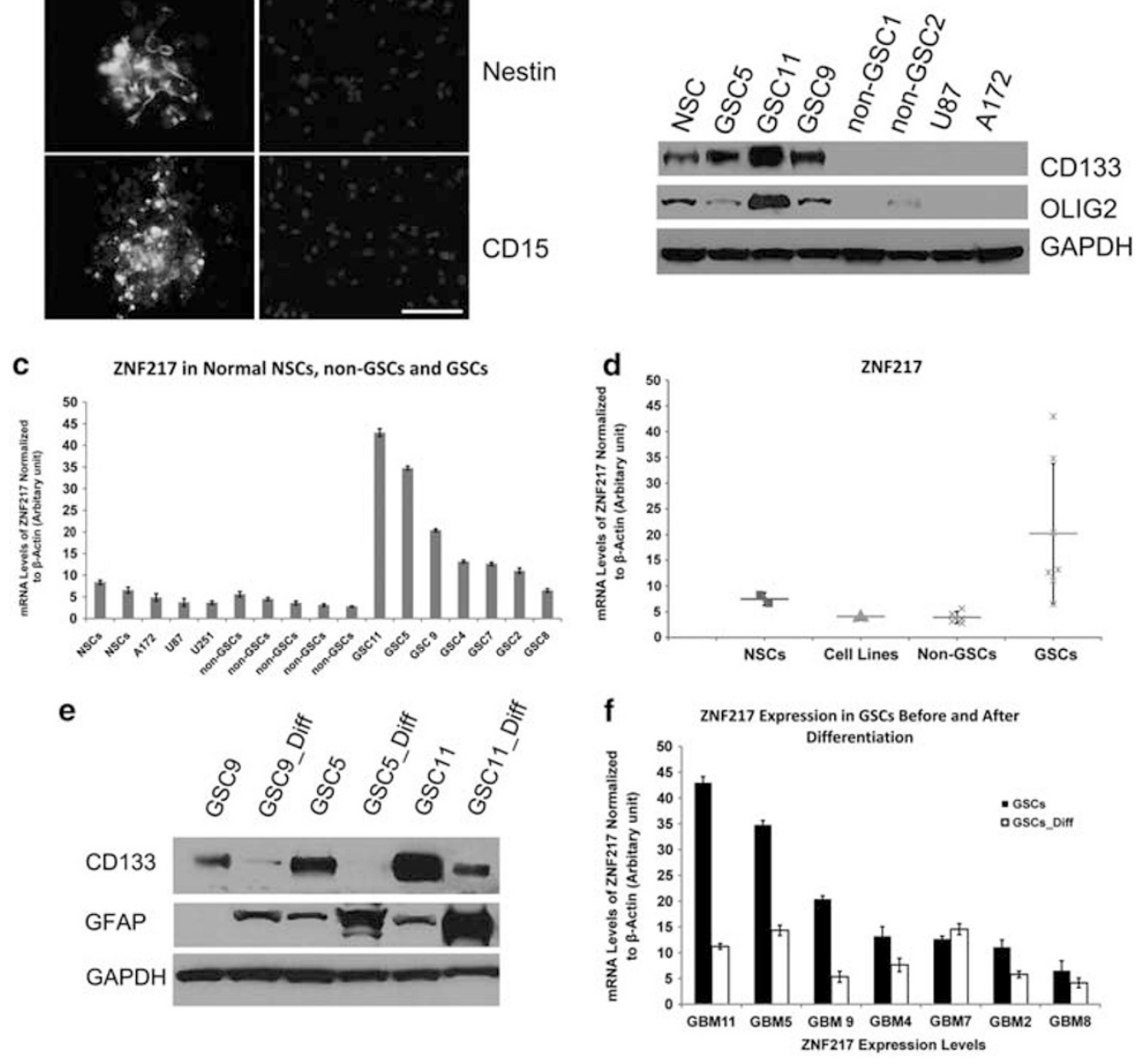

Figure 4 GSCs are enriched in ZNF217 compared with non-GSCs and normal NSCs. (a, b) Representative immunofluorescence images and western blots showing the expression of stem cell markers, Nestin, CD15, CD133 and OLIG2, in NSCs and GSCs, while little or no expression was seen in serum-cultured primary glioma cells and GBM cell lines (U87 and A172). (c, d) ZNF217 expression in normal NSCs, serum-cultured GBM cell lines, non-GSCs and GSCs. ZNF217 is overexpressed in GSCs compared with normal NSCs and non-GSCs. NSCs also expressed slightly higher levels of ZNF217 than non-GSCs.

(e) Representative western blots showing that the stem cell marker CD133 was reduced, while the glial marker GFAP was increased in differentiated GSCs. (f) qPCR showing ZNF217 expression was decreased in differentiated GSCs.

proliferative potentials of GSCs, we investigated the effects of ZNF217 knockdown in GSCs. The results showed that knockdown of ZNF217 inhibited the proliferation of GSCs and reduced Nestin-positive stem-like cell populations, implying that ZNF217 has a role in the maintenance of stem cells and inhibition of their differentiation.

Interestingly, ZNF217 is regulated by hypoxia, a critical factor for the GSC niche., ${ }^{911}$ Hypoxia is a well-recognized tumor microenvironmental factor that is linked to poor patient outcome and resistance to therapies. ${ }^{13,15,16}$ Therefore, it seems hypoxia has a fundamental role in the pluripotency and tumorigenicity of tumor stem cells; however, the mechanisms underlying its role in these two functions is not completely clear. HIFs have a central role in hypoxia-induced responses, as they can regulate many downstream target genes that promote stemness and tumorigenicity of tumor cells, such as VEGF, Notch, OCT4, hTERT and c-myc. Our results suggest that ZNF217 is regulated by HIFs, which is supported by the following evidence: (1) ZNF217 is positively correlated with HIF $1 \alpha$ and HIF2 $\alpha$ in GBM samples by qPCR; (2) knockdown of either HIF1 $\alpha$ or HIF $2 \alpha$ downregulates ZNF217 expression in GSCs and GBM cell lines; (3) ZNF217 is increased under hypoxia, and this hypoxia-induced increase is compromised when GSCs are treated with siRNA targeting HIF $1 \alpha$ or HIF $2 \alpha$. Together with our results showing that ZNF217 contributes to the maintenance of GSCs and inhibits their differentiation, these results suggest that ZNF217 may be a downstream target of HIFs that promotes the hypoxia-induced stemness of GSCs. Further exploring how HIFs regulate ZNF217 would provide more insight into the mechanisms of hypoxia-induced responses of GSCs. Genome-wide analysis of HIFs-binding site indicated that both HIF $1 \alpha$ and HIF $2 \alpha$ could bind to the promoter region of ZNF217, implying potential associations between HIFs and ZNF217. ${ }^{47}$ However, the binding regions of the HIFs do not contain the core hypoxia response element motif RCGTG, 


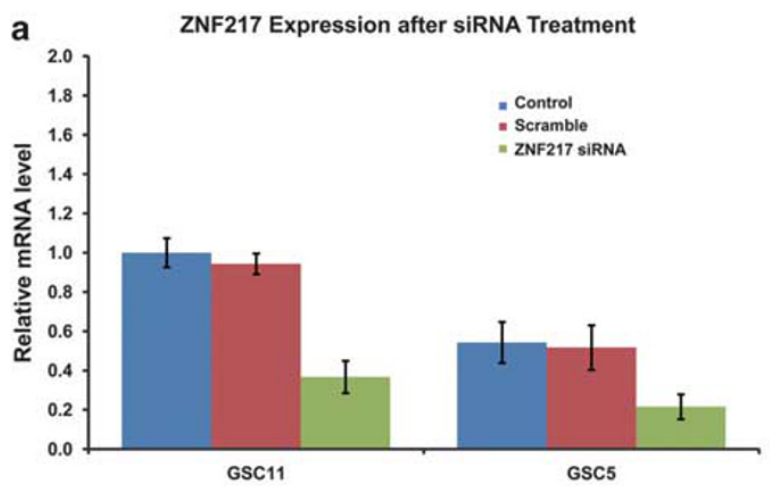

b

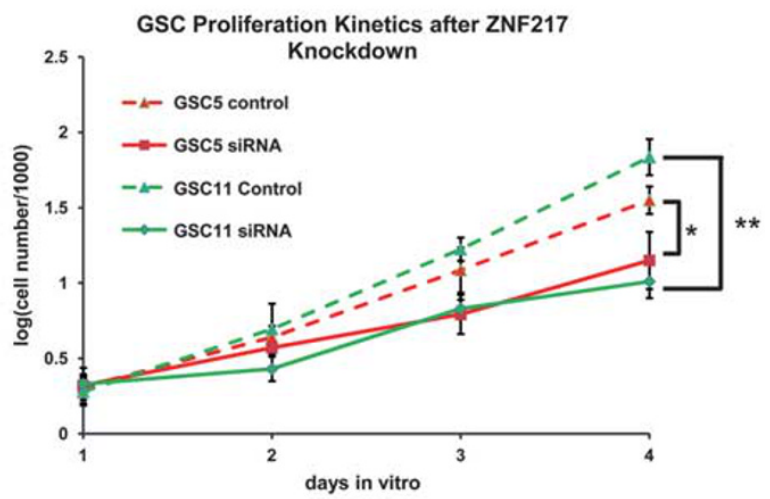

d

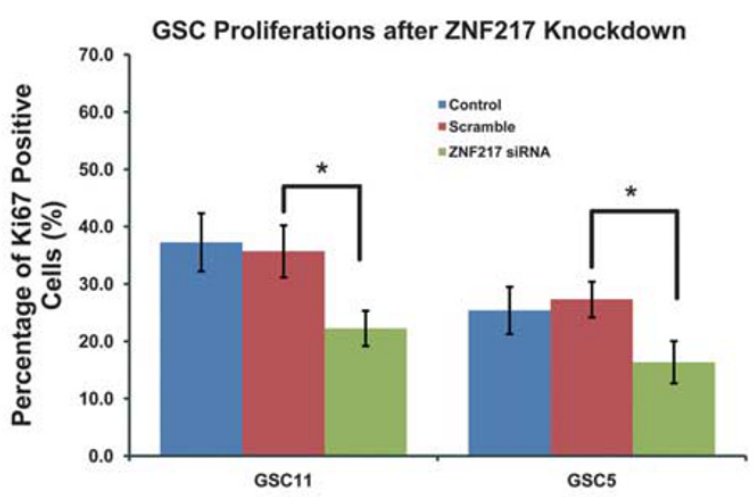

e

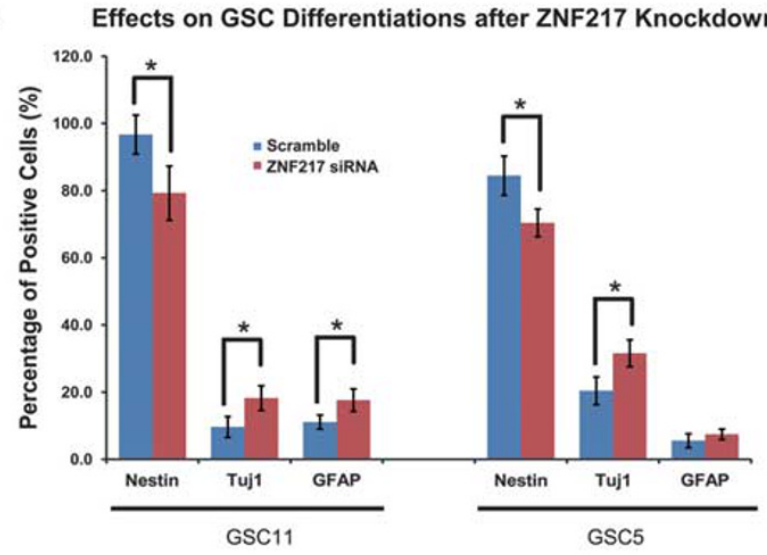

Figure 5 ZNF217 knockdown inhibited GSC proliferation and reduced the fraction of stem-like cells. (a) qPCR showing decreased ZNF217 expression in GSCs treated with ZNF217 siRNA. (b) ZNF217 siRNA inhibited GSC growth compared with non-targeting control siRNA as revealed by growth curves. (c) Representative images of immunofluorescence staining with Ki67, Nestin, Tuj1 and GFAP in GSCs after non-targeting control siRNA (left) or ZNF217 siRNA treatment (right). (d) Quantification of Ki67-positive cells indicates that proliferation was inhibited in GSCs by ZNF217 siRNA. (e) Quantification of immunofluorescence staining exhibited that ZNF217 siRNA reduced Nestin-positive stem-like cells and increased Tuj1-positive and GFAP-positive differentiated cells. 'Control' indicates parent cell line; 'scramble' indicates GSCs treated with non-targeting scramble siRNA.

suggesting that HIFs may bind the ZNF217 promoter indirectly or to other regions which are not detected by ChIP analysis. ${ }^{47}$

It has been reported that HIF $2 \alpha$ and multiple HIF-regulated genes are preferentially expressed in GSCs in compar- ison with non-GSCs. ${ }^{9,11}$ In tumor specimens, HIF2 $\alpha$ colocalizes with GSC markers and correlates with poor glioma patient survival. Notably, HIF $2 \alpha$ rather than HIF $1 \alpha$ is expressed over a wide range of oxygen levels and can provide GSCs a growth advantage by activating downstream targets 

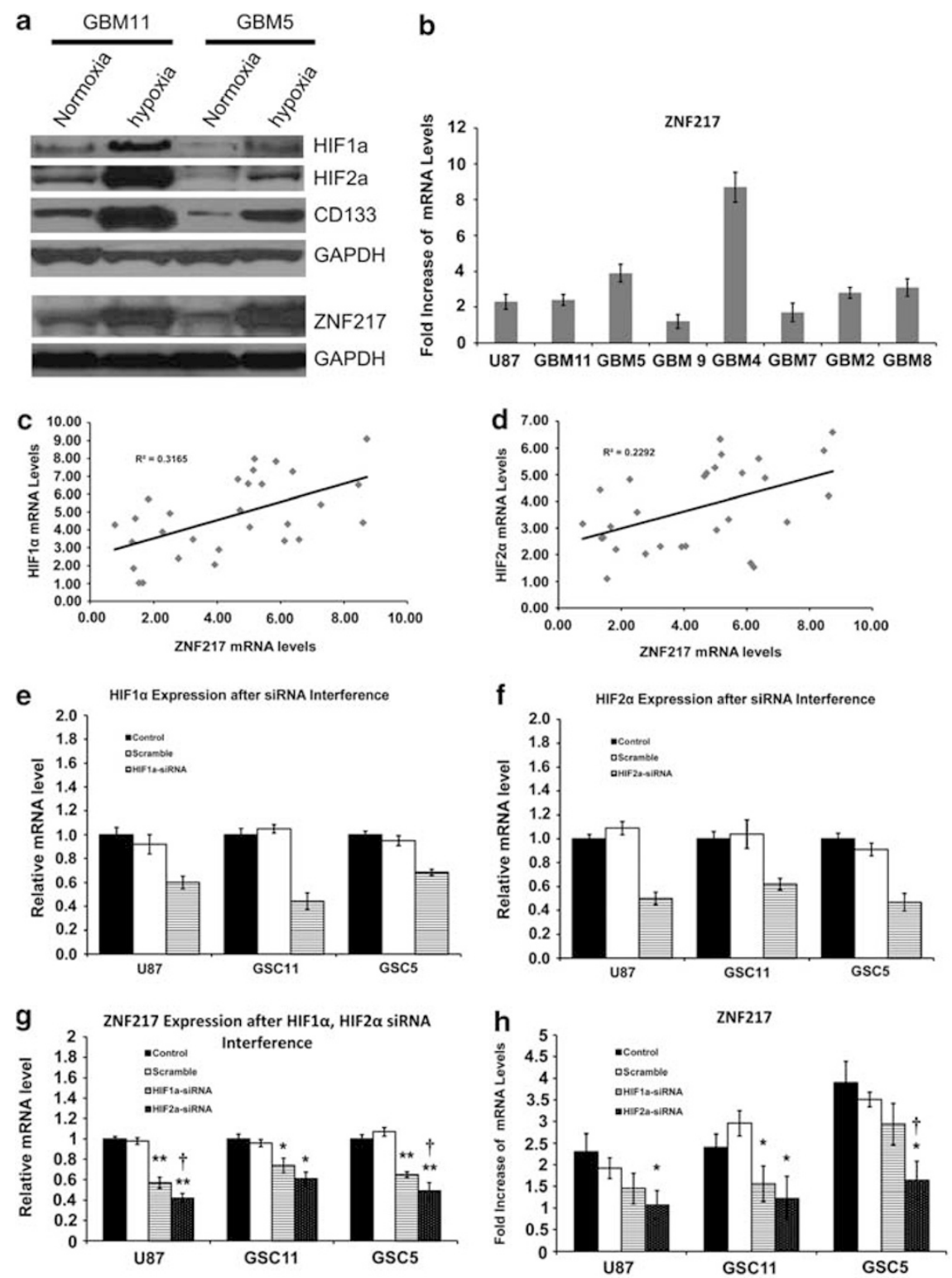

Figure 6 ZNF217 is increased under hypoxia and regulated by HIF1 $\alpha$ and HIF2 $\alpha$. (a) Representative western blots exhibiting HIF1 $\alpha$, HIF2 $\alpha$, CD133 and ZNF217 were increased under $1 \% \mathrm{O}_{2}$ hypoxic conditions for $24 \mathrm{~h}$ in GSC cells. (b) Fold-increase of ZNF217 mRNA levels in U87 and GSCs after exposing to hypoxia for $24 \mathrm{~h}$. (c, d) Correlation analysis of ZNF217, HIF1 $\alpha$ and HIF2 $\alpha$ expression in GBM samples by qPCR demonstrated that ZNF217 is significantly correlated with HIF $1 \alpha$ and HIF $2 \alpha(P<0.01)$. (e, f) qPCR showing efficiencies of HIF1 $\alpha$ and HIF2 $\alpha$ knockdown by siRNA in U87, GSC11 and GSC5. (g) ZNF217 expression was reduced in U87 and GSCs after HIF1 $\alpha$ or HIF2 $\alpha$ knockdown. ${ }^{\star} P<0.05,{ }^{*} P<0.001$ compared with non-targeting scramble control; ${ }^{\dagger} P<0.05$ compared with HIF1 $\alpha$ knockdown. (h) Fold-increase of ZNF217 under hypoxia demonstrated that ZNF217 upregulation was compromised in U87 and GSCs under hypoxia after HIF $1 \alpha$ or HIF $2 \alpha$ were knocked down in these cells. ${ }^{*} P<0.05,{ }^{* *} P<0.001$ compared with the non-targeting scramble control; ${ }^{\dagger} P<0.05$ compared with HIF1 $\alpha$ knockdown. qPCR data were normalized to $\beta$-actin with the levels of control in each group as 1 unit in (e-g); 'Control' indicates parent cell line; 'scramble' indicates GSCs treated with non-targeting scramble siRNA.

even without hypoxia. ${ }^{9}$ Therefore, it seems that HIF2 $\alpha$ specifically induces its effects on GSCs, and would be a promising target for anti-glioblastoma therapies. Intriguingly, our results indicated that HIF2 $\alpha$ regulates ZNF217 more efficiently than HIF1 $\alpha$ does. In addition, ZNF217 is more highly expressed in GSCs than in normal NSCs and non-GSCs (Figure $4 \mathrm{a}$ and $\mathrm{b}$ ), similar to the expression profile of HIF $2 \alpha{ }^{9}$ These data indicate that HIF $2 \alpha$ may activate
ZNF217 in GSCs to maintain the population even in the absence of hypoxia.

The mechanisms underlying ZNF217-induced maintenance of GSCs need to be further investigated, which would provide more insights into the intrinsic molecular basis controlling the biological features of GSCs. ZNF217 is believed to be a DNA-binding protein, which can function in gene expression by physically interacting with corepressor 
C-terminal-binding protein, ${ }^{48}$ histone deacetylases, histone methyltransferases and other proteins associated with transcriptional repressors. ${ }^{49-51}$ Overexpression of ZNF217 may provide a selective advantage to tumor cells by attenuating apoptotic events and by interfering with other pathways such as those associated with normal regulation of growth, death or differentiation. ${ }^{20,52}$ Therefore, ZNF217 may take its effects on GSCs by transcriptionally regulating downstream targets involved in tumorigenicity and differentiation. Identification of downstream targets of ZNF217 in GSCs may provide more comprehensive understanding of ZNF217 functions and reasonable strategies targeting ZNF217 in GSCs.

\section{DISCLOSURE/CONFLICT OF INTEREST}

The authors declare no conflict of interest.

1. DeAngelis LM. Brain tumors. N Engl J Med 2001;344:114-123.

2. Stupp R, Mason WP, van den Bent MJ, et al. Radiotherapy plus concomitant and adjuvant temozolomide for glioblastoma. N Engl J Med 2005;352:987-996.

3. Singh SK, Hawkins C, Clarke ID, et al. Identification of human brain tumour initiating cells. Nature 2004;432:396-401.

4. Lapidot T, Sirard C, Vormoor J, et al. A cell initiating human acute myeloid leukaemia after transplantation into SCID mice. Nature 1994;367:645-648.

5. Vescovi AL, Galli R, Reynolds BA. Brain tumour stem cells. Nat Rev Cancer 2006;6:425-436.

6. Mao XG, Zhang X, Zhen HN. Progress on potential strategies to target brain tumor stem cells. Cell Mol Neurobiol 2009;29:141-155.

7. Bao $\mathrm{S}, \mathrm{Wu} \mathrm{Q}, \mathrm{McLendon} \mathrm{RE}$, et al. Glioma stem cells promote radioresistance by preferential activation of the DNA damage response. Nature 2006;444:756-760.

8. Bar EE, Lin A, Mahairaki $\mathrm{V}$, et al. Hypoxia increases the expression of stem-cell markers and promotes clonogenicity in glioblastoma neurospheres. Am J Pathol 2010;177:1491-1502.

9. Li Z, Bao $S, W u$ Q, et al. Hypoxia-inducible factors regulate tumorigenic capacity of glioma stem cells. Cancer Cell 2009;15:501-513.

10. Calabrese $\mathrm{C}$, Poppleton $\mathrm{H}$, Kocak $\mathrm{M}$, et al. A perivascular niche for brain tumor stem cells. Cancer Cell 2007;11:69-82.

11. Seidel S, Garvalov BK, Wirta V, et al. A hypoxic niche regulates glioblastoma stem cells through hypoxia inducible factor 2 alpha. Brain 2010;133:983-995.

12. Mazumdar J, O'Brien WT, Johnson RS, et al. $\mathrm{O}(2)$ regulates stem cells through Wnt/beta-catenin signalling. Nat Cell Biol 2010;12:1007-1013.

13. Keith B, Simon MC. Hypoxia-inducible factors, stem cells, and cancer. Cell 2007;129:465-472.

14. Platet N, Liu SY, Atifi ME, et al. Influence of oxygen tension on CD133 phenotype in human glioma cell cultures. Cancer Lett 2007;258: 286-290.

15. Bertout JA, Patel SA, Simon MC. The impact of $\mathrm{O}_{2}$ availability on human cancer. Nat Rev Cancer 2008;8:967-975.

16. Pouyssegur J, Dayan F, Mazure NM. Hypoxia signalling in cancer and approaches to enforce tumour regression. Nature 2006:441:437-443.

17. Semenza GL. Life with oxygen. Science 2007;318:62-64.

18. Kaelin Jr WG, Ratcliffe PJ. Oxygen sensing by metazoans: the central role of the HIF hydroxylase pathway. Mol Cell 2008;30:393-402.

19. Mendez O, Zavadil J, Esencay M, et al. Knock down of HIF-1alpha in glioma cells reduces migration in vitro and invasion in vivo and impairs their ability to form tumor spheres. Mol Cancer 2010;9:133.

20. Krig SR, Miller JK, Frietze S. et al. ZNF217, a candidate breast cancer oncogene amplified at 20q13, regulates expression of the ErbB3 receptor tyrosine kinase in breast cancer cells. Oncogene 2010;29:5500-5510.

21. Sun G, Zhou J, Yin A, et al. Silencing of ZNF217 gene influences the biological behavior of a human ovarian cancer cell line. Int J Oncol 2008;32:1065-1071.
22. Quinlan KG, Verger $A$, Yaswen $P$, et al. Amplification of zinc finger gene 217 (ZNF217) and cancer: when good fingers go bad. Biochim Biophys Acta 2007:1775:333-340.

23. Li P, Maines-Bandiera S, Kuo WL, et al. Multiple roles of the candidate oncogene ZNF217 in ovarian epithelial neoplastic progression. Int J Cancer 2007;120:1863-1873.

24. Nonet GH, Stampfer MR, Chin K, et al. The ZNF217 gene amplified in breast cancers promotes immortalization of human mammary epithelial cells. Cancer Res 2001;61:1250-1254.

25. Collins C, Rommens JM, Kowbel D, et al. Positional cloning of ZNF217 and NABC1: genes amplified at 20q13.2 and overexpressed in breast carcinoma. Proc Natl Acad Sci USA 1998;95:8703-8708.

26. Krig SR, Jin VX, Bieda MC, et al. Identification of genes directly regulated by the oncogene ZNF217 using chromatin immunoprecipitation (ChIP)-chip assays. J Biol Chem 2007;282: 9703-9712.

27. Cowger JJ, Zhao Q, Isovic M, et al. Biochemical characterization of the zinc-finger protein 217 transcriptional repressor complex: identification of a ZNF217 consensus recognition sequence. Oncogene 2007;26:3378-3386.

28. Mao XG, Zhang $X, X u e X Y$, et al. Brain tumor stem-like cells identified by neural stem cell marker CD15. Transl Oncol 2009;2:247-257.

29. Cancer Genome Atlas Research Network. Comprehensive genomic characterization defines human glioblastoma genes and core pathways. Nature 2008;455:1061-1068.

30. Lee J, Kotliarova $S$, Kotliarov $Y$, et al. Tumor stem cells derived from glioblastomas cultured in bFGF and EGF more closely mirror the phenotype and genotype of primary tumors than do serum-cultured cell lines. Cancer Cell 2006;9:391-403.

31. Mao XG, Guo G, Wang P, et al. Maintenance of critical properties of brain tumor stem like cells after cryopreservation. Cell Mol Neurobiol 2010;30:775-786.

32. Soeda A, Park $M$, Lee $D$, et al. Hypoxia promotes expansion of the CD133-positive glioma stem cells through activation of HIF-1alpha. Oncogene 2009;28:3949-3959.

33. Heddleston JM, Li Z, McLendon RE, et al. The hypoxic microenvironment maintains glioblastoma stem cells and promotes reprogramming towards a cancer stem cell phenotype. Cell Cycle 2009;8:3274-3284.

34. Jordan CT. Cancer stem cells: controversial or just misunderstood? Cell Stem Cell 2009;4:203-205.

35. Chen R, Nishimura MC, Bumbaca SM, et al. A hierarchy of self-renewing tumor-initiating cell types in glioblastoma. Cancer Cell 2010;17: 362-375.

36. Beier D, Hau $P$, Proescholdt $M$, et al. CD133(+) and CD133(-) glioblastoma-derived cancer stem cells show differential growth characteristics and molecular profiles. Cancer Res 2007;67:4010-4015.

37. Son MJ, Woolard K, Nam DH, et al. SSEA-1 is an enrichment marker for tumor-initiating cells in human glioblastoma. Cell Stem Cell 2009;4:440-452.

38. Verhaak RG, Hoadley KA, Purdom E, et al. Integrated genomic analysis identifies clinically relevant subtypes of glioblastoma characterized by abnormalities in PDGFRA, IDH1, EGFR, and NF1. Cancer Cell 2010;17:98-110.

39. Phillips HS, Kharbanda $S$, Chen R, et al. Molecular subclasses of highgrade glioma predict prognosis, delineate a pattern of disease progression, and resemble stages in neurogenesis. Cancer Cell 2006;9:157-173.

40. Lottaz C, Beier D, Meyer K, et al. Transcriptional profiles of CD133+ and CD133- glioblastoma-derived cancer stem cell lines suggest different cells of origin. Cancer Res 2010;70:2030-2040.

41. Zheng $\mathrm{H}$, Ying $\mathrm{H}$, Wiedemeyer $\mathrm{R}$, et al. PLAGL2 regulates Wnt signaling to impede differentiation in neural stem cells and gliomas. Cancer Cell 2010;17:497-509.

42. Fan $\mathrm{X}$, Matsui $\mathrm{W}$, Khaki $\mathrm{L}$, et al. Notch pathway inhibition depletes stem-like cells and blocks engraftment in embryonal brain tumors. Cancer Res 2006;66:7445-7452.

43. Bar EE, Chaudhry A, Lin A, et al. Cyclopamine-mediated hedgehog pathway inhibition depletes stem-like cancer cells in glioblastoma. Stem Cells 2007;25:2524-2533.

44. Facchino S, Abdouh M, Chatoo W, et al. BMI1 confers radioresistance to normal and cancerous neural stem cells through recruitment 
of the DNA damage response machinery. J Neurosci 2010;30: 10096-10111.

45. Abdouh M, Facchino $\mathrm{S}$, Chatoo $\mathrm{W}$, et al. BMI1 sustains human glioblastoma multiforme stem cell renewal. J Neurosci 2009;29: 8884-8896.

46. Piccirillo SG, Reynolds BA, Zanetti N, et al. Bone morphogenetic proteins inhibit the tumorigenic potential of human brain tumourinitiating cells. Nature 2006;444:761-765.

47. Mole DR, Blancher C, Copley RR, et al. Genome-wide association of hypoxia-inducible factor (HIF)-1alpha and HIF-2alpha DNA binding with expression profiling of hypoxia-inducible transcripts. J Biol Chem 2009:284:16767-16775
48. Quinlan KG, Nardini M, Verger A, et al. Specific recognition of ZNF217 and other zinc finger proteins at a surface groove of C-terminal binding proteins. Mol Cell Biol 2006:26:8159-8172.

49. Shi YJ, Matson C, Lan F, et al. Regulation of LSD1 histone demethylase activity by its associated factors. Mol Cell 2005;19:857-864.

50. Chinnadurai $G$. Transcriptional regulation by C-terminal binding proteins. Int J Biochem Cell Biol 2007;39:1593-1607.

51. Banck MS, Li S, Nishio $\mathrm{H}$, et al. The ZNF217 oncogene is a candidate organizer of repressive histone modifiers. Epigenetics 2009:4:100-106.

52. Huang G, Krig S, Kowbel D, et al. ZNF217 suppresses cell death associated with chemotherapy and telomere dysfunction. Hum Mol Genet 2005;14:3219-3225. 